\title{
STUDIES AND RESEARCHES CONCERNING THE USE OF POROUS RESTRICTOR IN THE CASE OF HYDROSTATIC GUIDEWAYS
}

\author{
PASCU, M[arius]; OBREA, C[laudiu] F[lorin]; ANDRIOAIA, D[ragos] \& FUNARU, M[arian]
}

\begin{abstract}
The increase of manufacturing accuracy on the machine tools depends mainly on the motion accuracy of the mobile elements as: rams, mobile tables, pads, etc., the accuracy that can be achieved, firstly, by minimization of the friction in the guide ways. Nowadays, this problem is solved by replacing the sliding friction with the rolling friction buy especially by using the hydrostatic guide ways, where the contact surfaces are separated by a continuos film of fluid supplied by a pressure. In this paper such recent constructive solutions of guide way are presented. The presented guide ways have as principal components made by porous materials. The use of the porous restrictors becomes a practical necessity due to the fact that the advantage of its applying are multiple as: reduction of the friction force, reduction of the required energy necessary to moves the mobile elements the wear reduction and the improvement of the working conditions. The paper presents a simple guide way system that contains a porous restrictor that may be successfully applied in multiple applications in the field of machine tool.
\end{abstract}

Keywords: porous restrictor, hydrostatic guideway, pressure, viscosity, machine tools, portant force

\section{INTRODUCTION}

The increase and diversification of production on the machine tools equipped with bearings, hydrostatic guide ways and screws requires a careful study of these systems. On the other hand, the development of some non-conventional technologies for cold forming and the design of some automatic manufacture systems, leads to the necessity of the knowledge of design principles and norms of hydrostatic systems that are included in the above mentioned machine tools. The use of hydrostatic guideways in machine tools manufacture gives it superior performance, with direct effects on the quality of parts obtained by cutting [1]. The machine tools equipped with hydrostatic guide ways are more reliable, have very high productivity, and, not least, become more competitive on the international market. In the machine tools, the gideways are the elements that are the most subjected on the distructive action of friction [2]. It is known that in order to minimize the unwanted effect of this phenomenon, but always present the elements in contact and relative motion, it is necessary to use liquid or gaseous fluids. The problem that occurs in the case of hydrostatic guide ways is to maintain the thickness of fluid at a constant value when the external loadings present the important variation [3]. This involves the use of a single constant flow pumps to feed multiple hydrostatic pockets, with the condition that on each circuit is mounted a hydraulic resistance as restrictor or throttle.
The fixed supply device named throttle, restrictor or compensatory element, represent an important component in the supply system with lubrifiant in the case of a hidrostatic guideway. The use of compensatory elements leads to mantain the pressure at a constant value, being necessary when are supplied more pokets from a single pump. Xiaofeng, Lin and Taiyong have numerically analysed the aerostatic guideways equiped with porous restrictors by using finite element method. They have developed a new procedure for analysis and improvement of porous compensating elements. P. Tan et. al. have studied the porous restrictors from $\mathrm{Al}$ and $\mathrm{Ti}$ alloy particles. The results shows that the maximum pore size is increased with the increase of Al powder particle size, porosity and permeability is increased only when the particle size of $\mathrm{Al}$ is larger $-400 / 600$ of the mesh network. Durazo-Cardenas examines the porous restrictors made by ceramic materials used in the construction of hydrostatic guideways. The researches shows that use of these types of restrictors represents an improved alternative of the hydrostatic guideways due to its high stifness, low price and due to the fact that, in service, its generatea low tempeatures. By taking into account those above mentioned it can be conclude that the porous restrictors are used in the hydrostatic and aerostatic guideways. The aplications of the porous restrictors are rarely showed in the literature and the problem of their use is not enough studied. This paper presents a simple guideway system using the porous restrictor as a technical solution that can be applied successfully in the field of machine tools, especially for hydrostatic guideways.

\section{THE FIXED SUPPLY DEVICES}

The introduction of the compensatory elements in the presure supply scheme is equivalent to the use of an suplimentary hydraulic resistance in the fluid circuit. In Figure 1, the working principle of the compensatory elements is presented. It divides the total pressure drop between the power source with constant pressure $p_{a}$ and the external environment as follows:

$$
\Delta p=p_{a}-p_{0}=\left(p_{a}-p_{b}\right)+\left(p_{b}-p_{0}\right)
$$

where $\Delta p$ - the pressure drop; $p_{a}$-supply pressure, $p_{b}-$ pressure in pocket; $p_{o}$ - pressure in guide way. If the external loading is increased, the fluid film thickness is decreased, that leads to the decrease of the terms $p_{a}-p_{b}$, in other words, the increase of the pressure $p_{b}$ in pocket. For this reason it can be conclude that the used compensatory element must be a hydraulic resistance for which $\Delta p$ is a 
function of the flow rate developed in the mentioned element.

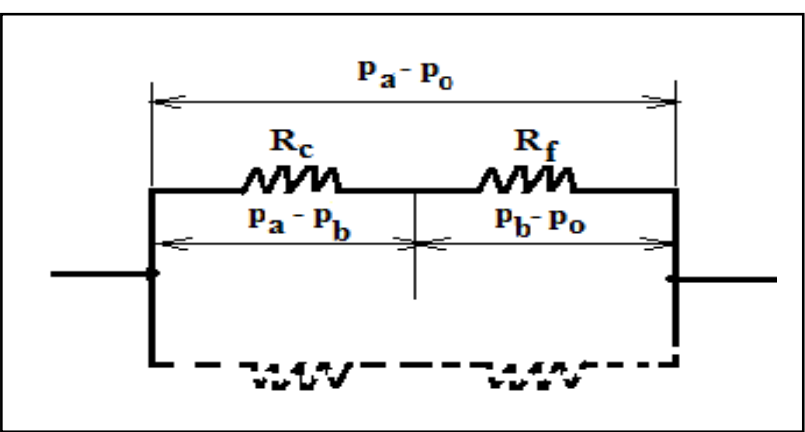

Fig. 1. Working principle of supply with the constant pressure

The main constructive and functional solutions of restrictors are as follows:

a) linear restrictors - in this case the flow rate equation have the general form as follows:

$$
Q_{r}=\frac{k_{r}}{\mu}\left(p_{a}-p_{b}\right)
$$

where, $k_{r}-$ the constant of restritor, $\mu-$ the dynamic viscosity of oil, $p_{a}$ - supply pressure and $p_{b}$ - pressure in poket.

b) nonlinear restrictors- the flow rate is expressed by equaton:

$$
Q_{r}=\left(p_{a}-p_{b}\right)^{\alpha}
$$

where, $p_{a}$ - supply pressure, $p_{b}$ - pressure in poket and $\alpha$ - flow rate coefficient in it simplest form, an eample of restrictor is represented by the capilar tube having the dimensions $d_{c}$ and $l_{c}$. - Figure 2 . The flow regime in the cappilary is laminal. By applying the Hagen-Poiseuille relation the flow ratw can be obtained:

$$
Q_{c}=\frac{\pi d_{c}^{4}}{128 \mu l_{c}}
$$

where $d_{c}$-diameter of capilary , $\mu$-dynamic viscozity, $l_{c}$ - lenght of capilary.

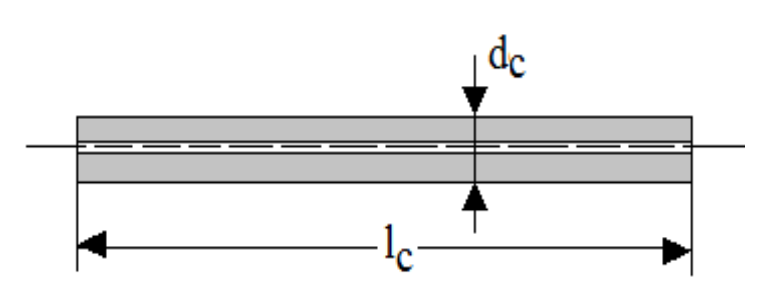

Fig. 2. Linear restrictor (capilary tube type)

The restrictors of the capillary tube type are characterized by simple design and less sensitive to temperature variation. As main disavantage are mentioned: risk of obstructing, require the ultrafiltration of the lubrificant high dimensions and slow performances for high loadings. In its simplest form, the nonlinear restrictors are represented by diaphragm - Figure 3. By comparing with the linear restrictors of capilary tube types it can be concluded that the nonlinear restrictors have some advantages as: superior performances espacially at high loadings, small dimensions and very compact. The main disavantages of the nonlinear restrictors are as follows: the calculation of the calibrated orifice is difficult and without accuracy, the characteristic of orifice is as a function of the manufacture accuracy; the risk of obstructing is mantained, an ultrafiltration of the lubrificant is required, the thickness of the film present an important variation with temperature. In order to prevent the risk of obstructing the dimensions of mesh will be $d>0.5 \mathrm{~mm}$. In the case of the orifice having the sharp edges, the flow rate vis given as follows:

$$
Q_{0}=k_{0} \sqrt{2} \sqrt{p_{a}-p_{b}}
$$

where $p_{a}$ - supply pressure, $p_{b}$ - pressure in poket, $k_{0}$ constant of orifice that is given by the relation:

$$
k_{0}=\propto_{Q} \cdot \pi \frac{d^{2}}{4} \cdot \frac{1}{\sqrt{\rho}}
$$

where $\alpha_{Q}$ - flow rate coeficient, $d$-diaphragm diameter, $\rho-$ oil density.

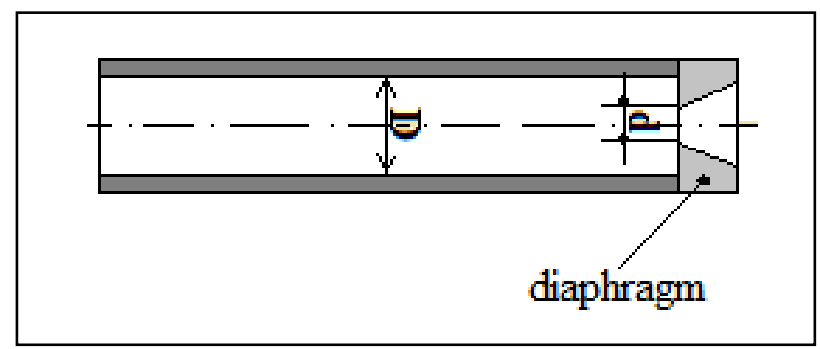

Fig. 3. Calibrated orifice restrictor type (diaphragm)

In practice, this type of restrictor is used in two concrete cases of top technology: hydrostatic guide ways for atomic electric power plant and for the rockets. From the above mentioned, it can be seen that this type of restrictor is recommended in those cases when the additional dimensional accuracy in the restrictor design do not represents an excesive cost by comparing with the assembly cost.

\section{THE POROUS RESTRICTOR}

As it is shown in Figure 4, a wide range of porous restrictors can be manufactured.

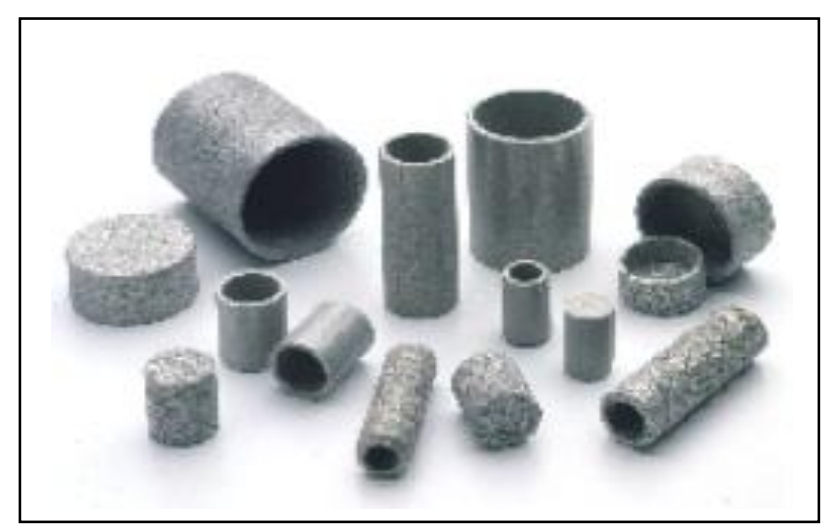

Fig. 4. Constructive solutions of porous restrictors

The porous restrictor is made by using a table of porous material that is mounted between the pressurs supply source and hydrostatic pocket as it is show i in Figure 5. It is known that permeability represents a complex geometric parameter, essential for characterizing of a porous environment. If the porous environment is 
modeled by the tangent spherical grains, the permeability can be calculated after Moscow, as follows:

$$
k_{p}=\frac{S_{3}^{2} d_{e}^{2}}{96(1-A)}
$$

where $S_{3}=S_{p} / S_{t}$, represents the ratio between total frontal surface of pore and the wetted surface by lubrificant; $A=V_{p} / V_{t}$, represents the ratio betwee the pore volume and total volume of tablet made by prous material. In the case of a maximum compactness the following relation is recomanded:

$$
k_{p}=1,215 \cdot 10^{-4} d_{e}^{2}
$$

where $d_{e}$ - the diameters of the spherical grains that are equals between. Due to the fact that this situation is hardly to obtain, it can be used the reccomanded value for the synterised metallic materials having the porosity between $20 \%$ and $45 \%$, namely $k_{p}=0.5\left(10^{-4}-10^{-12}\right) \mathrm{m}^{2}$. It can be observed that the range of $k_{p}$ is very large and, in this case, the experimental method remains the most reliable method in the determination of $k_{p}$.

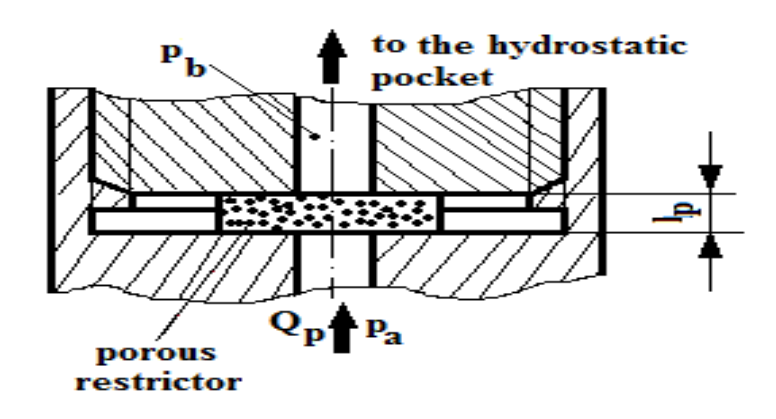

Fig. 5. Principle scheme of the porous restrictor

From the D'Arcy law, the flow rate can be calculated as follows:

$$
Q_{p}=\frac{\pi d_{p}^{2}}{4} \cdot \frac{k_{p}}{\mu} \cdot \frac{p_{a-} p_{b}}{l_{p}}
$$

where $d_{p}$ - diameter of particle, $k_{p}$ - permeability coefficient, $\mu$ - oil viscosity, $p_{a}$-supply pressure, $\mathrm{p}_{\mathrm{b}}-$ presure from hydrostatic pocket, $1_{p}-$ restrictor width. The change in prorosity due to partial clogging of the restrictor will influence the constant $k_{p}$ and will change the geometric constant of the restrictor.

\section{THE MATHEMATICAL MODELLING OF THE HYDROSTATIC GUIDEWAY SYSTEM WITH THE POROUS RESTRICTOR}

In Figure 6, the working principle of the hydrostatic guideway system equiped with the porous restrictor is schemetically presented. The fluid having supply pressure $p_{a}$ will pass through the porous material having the resistance $\rho_{l}$, reaching at a pressure $p_{j}$, for entry in the gap. In oprder to cross the entire gap, the fluid under pressure is supposed to a new pressure drop from $p_{j}$ to $p_{\text {atm }}$ (the air pressure). At the end of gap the fluid cross the resistance $\rho_{2}$. As a consequence if $p_{a}$ have a constant value, $p_{j}$ is influenced by two resistances: the porous metarial resistance $\rho_{1}$ and the plane gap resistance $\rho_{2}$. When the fluid under pressure are crossing in the porous material, between the contact surfaces a thin film having the thickness $h$ is created.

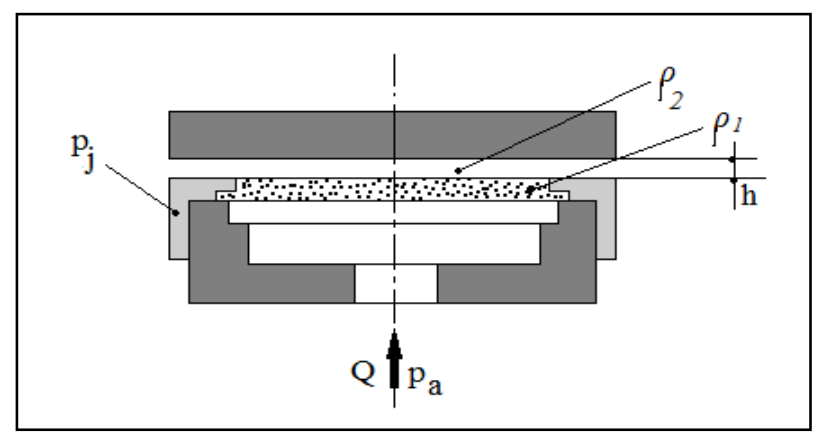

Fig. 6. Hydrostatic guideway system equiped with porous restrictor

At the same time a portant force proportional to entire surface of the porous restrictor will occurs. The portant force assure the sustentation of the mobile element of machine tool. Also, the portant force acts on the guideways. Due to the fact that thye gap in the porous restrictor are very small, it can be suppose that the fluid flow is laminar. As a consequence the D'Arcy law may be written as follows:

$$
V_{y}=\frac{d Q}{d S}=\frac{k_{p}}{\mu} \cdot \frac{\partial P}{\partial y}
$$

where : $V_{y}-$ is the fluid velocity on $y$ direction; $\mu-$ dynamic viscosity, $p$ - pressure, $k_{p}$ - permeability coefficient, $d Q$ - the flow rate in the element of surface $d S$, normal to direction $y$. in the case when the porosity of material are in range $20-60 \%$, the permeability coefficient $k_{p}$ has the values in range $0.5 \ldots 10^{-4}$. The loss of pressure on the distance $d_{y}$ can be determined from the above mentioned hypothesis, by using the Poiseuille relation as follows:

$$
d_{p}=\frac{32 \mu V_{y}}{d_{m}^{2}} d_{y}
$$

where: $d_{m}-$ is the maximum diameters of pores. The flow rate for a single pore is expressed as follows:

$$
Q_{1}=\frac{\pi}{4} \cdot d_{m}^{2} \cdot V_{y}
$$

From (11) and (12) another relation for the flow rate can be determined:

$$
Q_{1}=\frac{\pi d_{m}^{4}}{128 \mu} \cdot \frac{d P}{d y}
$$

If $N_{i}$, represents the number of orifices on the unitary surface, from the relation (13), values of flow rate which cross in the unitary surface of porous material can be determined as follows:

$$
d Q=N_{i} \cdot Q \cdot d s=\frac{N_{i} \cdot \pi d_{m}^{4}}{128 \mu} \cdot \frac{d p}{d y} \cdot d s
$$

In the case when the flow in the directions that are not normal to the element of surface is neglected, from (10) and (14) the following equation can be written:

$$
N_{i}=\frac{128 k_{p}}{\pi d_{i}^{4}}
$$

A porous restrictor having $N_{i}$ orifices on the element of surface is considered. It is known that the flow on the other direction than the normal direction to normal to 
surface is neglected if the thickness of the porous surface is sufficiently small. In the gap having the thickness $h$, the flow velocity of the fluid can be determined by using the Reynolds relation:

$$
U=\frac{1}{2 \mu} \cdot \frac{\partial P}{\partial x}\left(z^{2}-h z\right)
$$

where: U- velocity of fluid, $\mathrm{z}$ - the curent value of height, $\mathrm{h}-$ the thicknes of the lubrificant film, $\mu-$ dynamic viscosity.

The volumic flow rate is expressed by the following relation:

$$
Q=2 D \int_{0}^{h} u d z=\frac{D}{\eta} \cdot \frac{\partial P}{\partial x} \int_{0}^{h}(z-h z) d z
$$

The pressure variation in the gap for lubrification is given by the relation:

$$
d_{p}=\frac{12 Q}{D h^{3}} \eta d x
$$

By integrating (18) on obtain the expression of hydrostatic pressure distribution on the entire width of the guideway as follows:

$$
p^{2}=p_{j}^{2}+\left(p_{a t m}^{2}-p_{j}^{2}\right) \cdot \frac{x}{b}
$$

By integrating of the relation (19) for the entire surface of the guideway and substract the load that occurs due to external pressure, the carryng capacity can be obtained as follows:

$$
P=2 b\left[\frac{2\left(p_{j}^{3}-p_{a t m}^{3}\right)}{3\left(p_{j}^{2}-p_{a}^{2}\right)} N_{i} l-L p_{a t m}\right]
$$

where: $N_{i}$ - number of orifices, $L$ - the guideway lenght, $l$-distance between two orifices.

The pressure $p_{j}$, deppends on the porous restrictor resistance and on the thickness of gap. The guideway stifness can be calculated by applying the following relation

$$
J=8 b N_{i} l \cdot \frac{K_{0} p_{j}\left(p_{j}^{4}-3 p_{j}^{2}+2 p_{j}\right)}{h\left[p_{a t m}^{2}+K_{0}+\left(3 p_{j}^{4}-2 p_{j}^{2}-1\right)\right]}
$$

where: $K_{0}=f\left(p_{j}\right), K_{0}=f\left(p_{a} / p_{a t m}\right)-$ represents a functional paramater as a function of the supply pressure.

\section{IMPLEMENTATION OF THE MODEL AND RESULTS}

Availability and existence of multiple hardware devices, computers and mathematical simulation software, allows the testing and execution of the simulation operations even for very complicated systems. In order to implement the above presented mathematical model Matlab 2009b software was used.For different load levels and different dimensions of the porous restrictor as a function of the supply pressure $p_{a}$, a series of values for the thicknes of film lubrificant is obtained. From the analysis of the results presented in Figure 7 it can be concluded that for a certain value of the external loading of the guideway, the thickness of the lubrificant film must be constant.

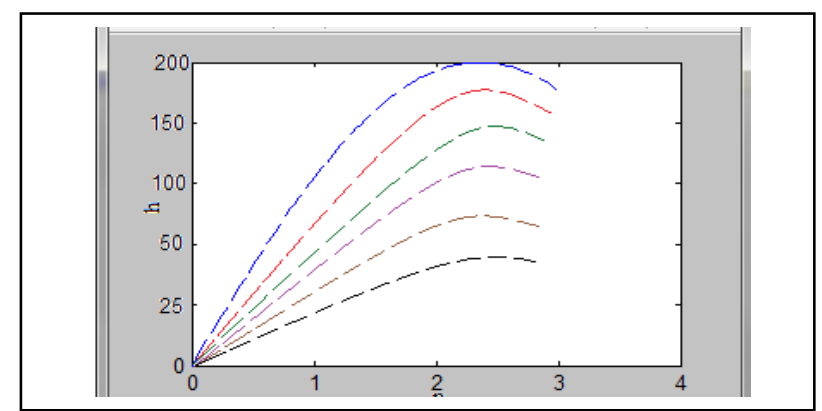

Fig. 7. Variation of the film lubrificant thickness $h$ as a function of supply pressure $p_{a}$

From the results show in Figure 8, it can be remarked the sensitivity of the film thickness $h$ with the increase of the external loading.

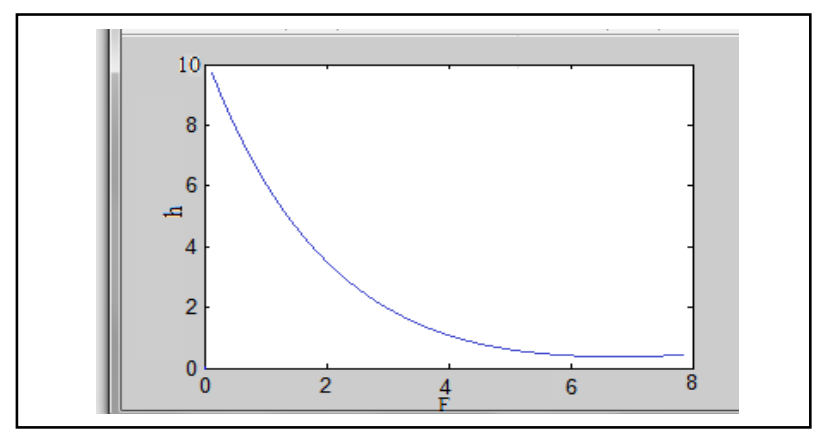

Fig. 8. Variation of the film thickness h, as a function of external loading $F$

\section{CONCLUSIONS}

This paper shows the posibility for use of porous restrictor on the hydrostatic guideways. A mathemetical model was developed that describing the behavior of a porous restrictor as part of a simple hydrostatic guideway. The results was obtained by implementing of the equations in Matlab 2009b software. From the characteristics obtained after model implementation it is remarked that the increase of the external loading leads to the decrease, in the unwanted manner, of the film thickness $h$. The modification of the porosity due to colgging of restrictor will influence the constant $k_{p}$ and will change the geometric constant of the restrictor.

\section{REFERENCES}

[1] Oh Y.J., Park C.H., Lee H. \& Lee D.W., (2004). Measurement of 5 DOF motion errors in the ultra precision feed tables for error compensation. Proceedings of KSPE Autumn Annual Meeting 2004, pp. 672-678

[2] Brecher C, Utsch P, Klar R \& Wenzel C., (2010). "Compact design for high precision machine tools". International Journal of Machine Tools and Manufacture, Vol. 50, No.4, pp. 328-334

[3] Graboskii, V I, (2006). "Optimum porous journal bearing ensuring maximum load capacity". Fluid Dynamics, Vol. 41, No.1, pp. 29-36

[4] Xiaofeng, Z., Lin, B., Taiyong, W., (2009). 'Finit element analyses of aerostatic lubrication with pourous restrictors based on HYPERMESH'. Journal of Vacuum and Technology, Vol. 27, No. 3, pp. 244-248

[5] Tan, P., Tang, X. \& Li, C., (2009). "Research on TiAl Alloy Porous Meral Flow Restrictors". Material Transaction, Vol. 50, No. 10, pp.2484-2487

[6] Cardenas, D., (2010). "The performance of a pourous ceramic hydrostatic journal bearing". Journal of Engineering Tribology, vol. 224, No.1, pp.81-89 